\title{
Princípios organizadores da representação social do envelhecimento: dados coletados via internet
}

\author{
João Fernando Rech Wachelke \\ Brigido Vizeu Camargo \\ Joana Vieira Hazan \\ Universidade Federal de Santa Catarina \\ Débora Ribeiro Soares \\ Universidade Federal da Paraíba \\ Luísa Todeschini Pereira Oliveira \\ Universidade Federal de Uberlândia \\ Paula Debiasi Reynaud \\ Universidade Federal de Santa Catarina
}

\begin{abstract}
Resumo
Foi realizada uma pesquisa com o objetivo de caracterizar os princípios organizadores da representação social de jovens e adultos não-idosos sobre envelhecimento. Houve participação de 469 indivíduos, que foram convidados a responder um instrumento que lhes pedia a evocação de 7 a 10 palavras sobre envelhecimento, por meio de mensagens divulgadas em comunidades de internet. Por meio de análises de correspondências com o programa Spad, foi identificado um princípio geral opondo perdas e ganhos, típico da representação sobre envelhecimento, e também os contrastes entre os grupos de diferentes faixas etárias, permitindo caracterizar a mudança do pensamento acerca de envelhecimento com o passar da idade. Discute-se também a necessidade de caracterizações estruturais mais precisas sobre a representação do envelhecimento.
\end{abstract}

Palavras-chave: representação social; envelhecimento; princípios organizadores; questionário online

\begin{abstract}
Social representation on aging's organizing principles: data collection via internet. A study was carried out with the goal of describing the organizing principles related to the social representation of young and nonelderly adult people about aging. A number of 469 individuals participated. They were invited to answer an instrument which asked them for 7 to 10 word evocations on aging, by means of messages spread through internet communities. Through correspondence analyses executed with the aid of the Spad software, a general principle opposing losses and gains was identified, which is typical of the representation on aging, and there were also contrasts among the groups of different age ranges, allowing for a description of the change in thought concerning aging with the passing of years. The need of more precise structural characterizations about the social representation on aging is also discussed.
\end{abstract}

Keywords: social representation; aging; organizing principles; online questionnaire

$\mathrm{O}$ envelhecimento populacional, fenômeno mundial segundo o qual os idosos tornam-se uma cota mais representativa no conjunto total da população (Kinsella \& Velkoff, 2001) sofre influência da diminuição de taxas de natalidade, diminuição da mortalidade por causas patológicas em populações adultas e aumento da expectativa de vida na velhice (Neri, 2001).

No Brasil, está em curso uma inversão acelerada da pirâmide populacional, ocasionada principalmente pelo declínio de taxas de fecundidade. Devido ao ritmo maior desse declínio e ao fato de este estar situado numa população mais jovem que a de países desenvolvidos, o processo de envelhecimento populacional tende a ser mais rápido e propiciar mudanças demográficas mais profundas (Carvalho \& Garcia, 2003). Estima-se que 9,9 milhões dos 169 e meio do Brasil, correspondente a 5,8\% da população, tivessem 65 anos ou mais durante o censo de 2000 (IBGE, 2001). $\mathrm{Na}$ Pesquisa Nacional por Amostras de Domicílios de 2005, essa proporção subiu para $6,8 \%$ da população (IBGE, 2006).

A psicologia social necessita levar em consideração os processos relacionados ao envelhecimento, que podem atuar em contextos cognitivos individuais e intergrupais. Segundo $\mathrm{Ng}$ (1998), é necessário fazer pesquisas sobre o que as gerações 
pensam e como agem umas em relação às outras. Cabe também aos psicólogos sociais a tarefa de identificar diferenças em processos sociopsicológicos com base nos tipos de relações implicadas entre atores sociais e mesmo características do processamento cognitivo desses atores. Estudar o conhecimento compartilhado sobre o processo de envelhecimento permite responder questões dessas variedades.

\section{Teoria das representações sociais}

Em psicologia social, o estudo das representações sociais, na perspectiva teórica inaugurada por Moscovici (1961/1976), teve início nos anos 1960 e mostrou-se um paradigma de pesquisa capaz de explicar a natureza e transformação do senso comum e de diversos processos comunicacionais e comportamentais ligados a grupos sociais. Representação social designa ao mesmo tempo um produto e um processo (Valsiner, 2003). Enquanto processo, "é uma forma de conhecimento, socialmente elaborada e partilhada, com um objetivo prático, e que contribui para a construção de uma realidade comum a um conjunto social" (Jodelet, 2001, p. 22). O processo de representar resulta em teorias do senso comum, elaboradas e partilhadas socialmente (Wagner, 1998), ligadas a inserções específicas dentro de um conjunto de relações sociais, isto é, a grupos sociais (Doise, 1985), que têm por funções explicar aspectos relevantes da realidade, definir a identidade grupal, orientar práticas sociais e justificar ações e tomadas de posição depois que elas são realizadas (Abric, 1998).

Em relação ao funcionamento das representações sociais, Moscovici (1961/1976) identifica dois processos principais: objetivação e ancoragem. A objetivação é o processo por meio do qual uma noção abstrata ganha forma e torna-se concreta por meio de imagens ou idéias, resultando numa organização dos conteúdos semelhante a um mapa conceitual (Doise, Clemence, \& Lorenzi-Cioldi, 1992). A ancoragem é o processo de classificar informações sobre um objeto social em relação a estruturas de conhecimento anteriormente existentes, ligando as representações sociais a uma memória coletiva (Moscovici, 2003).

No presente caso, interessa o estudo do processo de ancoragem, para o qual a Escola de Genebra desenvolveu métodos adequados (Doise, Clemencea, \& Lorenzi-Cioldi, 1992). Trata-se de uma perspectiva não consensual das representações sociais, que são tomadas como princípios geradores de variações entre tomadas de posição de indivíduos. Esses princípios estão ligados a inserções específicas dentro de um conjunto de relações sociais e organizam os processos simbólicos que intervêm nessas relações (Doise, 1985). Assim, o estudo não enfoca propriamente estruturas representacionais objetivadas de um grupo, mas, em vez disso, dá ênfase aos pontos de referências que orientam o debate social, permitindo o posicionamento de grupos e indivíduos em diversas localidades desse "espaço" de conhecimento (Doise, Clemence, \& Lorenzi-Cioldi, 1992).

O conhecimento sobre representações sociais de grupos sobre envelhecimento e velhice pode fornecer dados para caracterizar suas produções simbólicas frente ao desenvolvimento da vida, situando relações frente a outros grupos de seu ambiente social. De modo complementar, estudar representações sociais de pessoas de grupos etários diferentes sobre algum objeto social pode ser útil para identificar diferenças de processamento da realidade específicas a esses grupos.

\section{Representações sociais sobre envelhecimento}

Nas pesquisas sobre percepções sociais e representações sociais são relevantes os estudos sobre as crenças acerca de objetos sociais relacionados ao envelhecimento: o envelhecimento propriamente dito, a velhice e o idoso. As pesquisas geralmente centram-se em populações de idosos e jovens, ou na análise da comunicação midiática.

Nos países mais desenvolvidos que o Brasil, o envelhecimento populacional está em estágio mais avançado. Porém, mesmo com maior participação dos idosos na sociedade, as pesquisas sobre o tema indicam predominantemente uma visão negativa.

Nos Estados Unidos, onde $12,6 \%$ da população possuía 65 anos ou mais de idade em 1975 (Kinsella \& Velkoff, 2001), Brewer, Dull e Lui (1981) e Hummert (1990) estudaram os estereótipos de idosos mantidos por estudantes universitários, deparando-se com imagens tanto positivas quanto negativas. No que diz respeito às atitudes, as pesquisas indicam que em relação aos idosos elas são piores que as em relação aos jovens, ainda que isso possa ser afetado por características da pesquisa (Kite \& Johnson, 1988).

Na Alemanha, onde $16,2 \%$ da população tinha 65 anos ou mais em 2000 (Kinsella \& Velfoff, 2001), Kessler, Rakoczy e Staudinger (2004) observaram que as pessoas idosas são sub-representadas na televisão. Também foi constatada a predominância de uma visão da velhice distante da realidade: os idosos retratados geralmente contraem poucas doenças, e assuntos como relações familiares, aposentadoria e redes sociais são pouco abordados.

Por fim, na Itália, onde a proporção de idosos é uma das maiores do mundo, com $18,1 \%$ da população do país tendo 65 ou mais anos de idade (Kinsella \& Velkoff, 2001), Gastaldi \& Contarello (2006) observaram que, para os jovens, o envelhecimento é um processo negativo de enfraquecimento. Para adultos não-idosos, envelhecimento está ligado a uma vida mais tranqüila. Os idosos possuem atitudes positivas sobre envelhecimento. Hubbard (2007) observou que nos anúncios publicitários predominam estereótipos negativos e representações não realistas dos idosos. Foi também verificada diferença na representação do idoso entre jovens, que representam-no com sabedoria e experiência, mas ligado à velhice e passividade; e idosos, que fornecem mais palavras positivas e falam de felicidade e família, mas também de doenças.

Num contexto brasileiro, Neri (1991) realizou uma pesquisa sobre atitudes com uma amostra representativa de não-idosos para o Brasil. Surpreendentemente, predominavam atitudes favoráveis acerca do idoso e da velhice para essa população.

Veloz, Nascimento-Schulze e Camargo (1999) investigaram as representações sociais do envelhecimento em idosos de Florianópolis. Foram identificadas três representações sociais diferentes: uma primeira, típica de mulheres, que se refere à velhice como perda da beleza e relações sociais e familiares; uma segunda trata da velhice como uma etapa em que se perde o ritmo de trabalho; e uma terceira representação, concebe 
o envelhecimento como um desgaste físico natural, menos importante que o desenvolvimento mental. Um estudo de Teixeira (2000) encontrou suporte empírico para o surgimento de alguns ganhos do envelhecimento: os mecanismos cognitivos que surgem para compensar as perdas do processo. Mais recentemente, Oliveira e Santos (2002), identificaram junto a funcionários aposentados de uma universidade brasileira também um eixo de ganhos e um eixo de perdas para a representação do velho.

Santos e Belo (2000) entrevistaram idosos dos meios rural e urbano nordestino, e chegaram a três modelos de velhice: uma velhice-experiência, típica do sertão rural, em que o idoso é valorizado; uma velhice-doença, modelo biológico que enfatiza aspectos de declínio, partilhado pelos participantes da zona urbana e, finalmente, uma velhice-reivindicação, uma visão de revalorização do idoso na zona urbana.

Martins (2002) entrevistou adolescentes, adultos e idosos, todos usuários de uma associação comercial, sobre o significado de idoso e velhice. Para ambas as representações há uma diferença nos conteúdos do pensamento de jovens e adultos não-idosos, de um lado, que concebem o idoso como uma pessoa dependente que deve ser respeitada por sua experiência, e a velhice como uma etapa ou fase natural da vida, um processo que gera dificuldade mas também resulta de muita experiência; e os idosos, que falam dessa etapa com naturalidade, abordando aspectos do cotidiano.

Araújo, Carvalho e Moreira (2003) entrevistaram idosos de grupos de convivência paraibanos e constataram que suas concepções de velhice e envelhecimento eram ligadas a elementos negativos, como demência, senilidade, solidão, rejeição familiar e preconceito. Araújo, Coutinho, Barros e Moreira (2005) verificaram que a representação social sobre a velhice para idosos de grupos de convivência paraibanos está associada a doença. Também junto a idosos de grupos institucionais, Costa e Campos (2003) verificaram que a representação social de velhice tem aspectos comuns como declínio e morte.

Mithidieri e Tura (2003) realizaram um estudo junto a estudantes de medicina do Rio de Janeiro e encontraram evidências de que o núcleo central da representação social do idoso para esse grupo seja o tema sabedoria. Mesmo assim, encontram-se muitos elementos com conotação negativa, como frágil, discriminado, doença e morte.

Ainda não há estudo feito com amostras brasileiras que busque identificar, junto a uma amostra de médio porte, além dos elementos da representação, os princípios organizadores na representação do envelhecimento compartilhada por atores sociais brasileiros de diferentes grupos geracionais e sócioeconômicos, viabilizando a identificação de pontos de ancoragens dos diversos grupos que compõem a sociedade brasileira acerca de como representar o envelhecimento. Em escala menor, tratase do que Gastaldi e Contarello (2006) realizaram junto a uma amostra do norte da Itália.

A presente investigação tem a finalidade de obter resultados nesse sentido. Seu objetivo principal é caracterizar o campo da representação social do envelhecimento para uma amostra brasileira, incluindo princípios organizadores que segmentem essa representação, ou representações, em termos de pontos de ancoragem sócio-grupais.

\section{Método}

\section{Participantes}

Participaram do estudo 469 indivíduos, todos usuários de um sítio de internet de relacionamentos. Deles, 55,8\% eram do sexo feminino. A média de idade dos participantes foi de 30 anos e 6 meses, com grande variabilidade, a saber pelo desvio padrão de 12 anos. A mediana da idade da amostra foi de 27 anos. As idades dos participantes variaram de 15 a 59 anos. O percentual de $25,6 \%$ declarou ter até 21 anos de idade, em seguida $32,8 \%$ disseram ter entre 22 e 29 anos, 26,9\% declararam possuir de 30 a 45 anos, e 14,7\%, mais de 45 anos. Foi observada uma quantidade muito baixa de respondentes com 60 anos ou mais (apenas 6) e, portanto, eles não foram considerados da pesquisa, não sendo computados no total de 469 da amostra. Quanto à região geográfica brasileira em que residiam os participantes, 39,2\% habitavam em cidades do sudeste, seguidos por moradores do sul $(22,8 \%)$ e nordeste $(19,6 \%)$. O estado com mais respondentes foi São Paulo (23\% do total), seguido por Santa Catarina $(13,2 \%)$ e Rio de Janeiro (6,6\%). A Tabela 1 apresenta as características dos participantes.

Em termos das regiões onde habitam, a amostra apresenta distorções quanto às proporções encontradas nas estimativas da Pesquisa Nacional por Amostras de Domicílios (PNAD) do Instituto Brasileiro de Geografia e Estatística (IBGE, 2006), identificando-se sub-representação dos participantes do nordeste e super-representação dos participantes do sul. Verificou-se predominância de participantes solteiros, com altos níveis de escolaridade, renda familiar elevada [segundo a PNAD, mais da metade dos domicílios brasileiros possui renda familiar de até 3 salários mínimos].

\section{Instrumento}

Foi utilizado um questionário com questões de caracterização dos participantes (sexo, idade, estado civil, escolaridade, estado de residência e renda familiar declarada) e uma tarefa de evocação livre. Pedia-se aos participantes que escrevessem de 7 a 10 palavras ou expressões que lhes viessem à consciência ao pensar em envelhecimento.

\section{Procedimento}

A coleta de dados foi realizada no segundo semestre de 2006. Um link de internet foi divulgado no site de relacionamentos Orkut da internet, uma das maiores redes online em comunidades virtuais, referentes a 150 cidades brasileiras de todas as regiões do país. Para tanto, foi criado um tópico em que havia um texto de introdução ao estudo, advertindo os internautas de que não se tratava de uma enquete comum de opinião divulgada pela internet, mas de um estudo científico sobre envelhecimento, convidando-os a participar..

Ao clicarem no link, os participantes acessavam um site da internet que apresentava a equipe de pesquisa e explicitava o modo de participação por meio do preenchimento do 
Tabela 1

Caracterização da amostra $(N=469)$

\begin{tabular}{|c|c|c|}
\hline Variáveis & Freqüência & $\%$ \\
\hline \multicolumn{3}{|l|}{ Sexo } \\
\hline Mas culino & 210 & 44,8 \\
\hline Feminino & 259 & 55,2 \\
\hline \multicolumn{3}{|l|}{ Estado civil } \\
\hline Solteiro & 305 & 65,0 \\
\hline Casado & 117 & 24,9 \\
\hline Separado / divorciado & 42 & 9,0 \\
\hline Viúvo & 5 & 1,1 \\
\hline \multicolumn{3}{|l|}{ Nível de escolaridade } \\
\hline Ensino fundamental & 9 & 1,9 \\
\hline Ensino médio & 82 & 17,5 \\
\hline Ensino su perior completo ou incompleto & 378 & 80,6 \\
\hline \multicolumn{3}{|l|}{ Faixa etária } \\
\hline Até 21 anos & 120 & 25,6 \\
\hline De 22 a 29 anos & 154 & 32,8 \\
\hline De 30 a 45 anos & 126 & 26,9 \\
\hline Mais de 45 anos & 69 & 14,7 \\
\hline \multicolumn{3}{|l|}{ Região de residência } \\
\hline Norte & 35 & 7,5 \\
\hline Nordeste & 92 & 19,6 \\
\hline Centro-Oeste & 51 & 10,9 \\
\hline Sudeste & 184 & 39,2 \\
\hline Sul & 107 & 22,8 \\
\hline \multicolumn{3}{|l|}{ Renda familiar declarada } \\
\hline Até $\mathrm{R} \$ 700$ & 19 & 4,1 \\
\hline De R\$ 701 a R\$ 1750 & 96 & 20,5 \\
\hline De R\$ 1751 a R\$ 3500 & 157 & 33,5 \\
\hline De R \$ 3501 a R\$ 7000 & 132 & 28,1 \\
\hline Mais de $\mathrm{R} \$ 7000$ & 65 & 13,9 \\
\hline
\end{tabular}

questionário. Os participantes foram informados acerca do sigilo dos dados e dos aspectos éticos do estudo, sendo convidados a confirmar sua participação clicando num botão para avançar à tela seguinte, deparando-se com o formulário eletrônico com o questionário.

O formulário continha instruções de programação segundo as quais os participantes recebiam uma mensagem na tela caso não tivessem preenchido todas as questões, indicando os itens de que eles haviam se esquecido, para evitar o envio de dados incompletos. Os dados enviados foram armazenados no servidor da internet, juntamente com informações relativas à data e hora de preenchimento e o endereço de IP dos computadores dos respondentes.

\section{Análise dos dados}

Antes de serem submetidos a análise estatística, os dados sofreram uma pré-análise, para remoção de questionários inválidos ou em excesso ocasionados por participantes que tivessem respondido a pesquisa mais de uma vez, deliberadamente ou não; para tanto foram observados os dados referentes a data e hora de preenchimento e endereço de IP (Protocolo de Internet), que orientou a exclusão de questionários repetidos, ou de questionários com dados diferentes em que constava o mesmo IP.

Em seguida, as palavras foram categorizadas tematicamente (Bardin, 1977). As categorias foram interpretadas como elementos da representação social do envelhecimento. O critério básico de agrupamento foi semântico.

Foram realizadas análises fatoriais de correspondência (Cibois, 1983; Cibois, 1989). A análise de correspondências é uma técnica exploratória e descritiva que tem por finalidade investigar a associação de variáveis nominais, podendo ser executada com respostas de evocações livres, sintetizando tabelas de contingência. Para pesquisadores em representações sociais, os fatores podem ser interpretados como princípios que organizam e explicitam diferenças da representação social para os grupos 
considerados, constituindo uma espécie de supra-estrutura, ideal para verificar variações no campo da representação incluindo indivíduos com características diferentes, ou pertencentes a grupos diferentes (Doise, Clemence, \& Lorenzi-Cioldi, 1992; Oliveira \& Amâncio, 2005). A análise foi realizada com o auxílio do programa $S P A D$, v. 6.

\section{Resultados}

As seguintes categorias, tratadas como elementos da representação, foram empregadas (número de ocorrências no corpus entre parênteses): aborrecimento (20), aceitação (13), afeto (46), aposentadoria (63), aspecto financeiro (26), atividades e hábitos (104), autonomia (34), cansaço (79), aspectos orgânicos (23), declínio (261), dependência (54), descanso (39), desprendimento (11), desvalorização social (71), direitos (18), disponibilidade, doenças (207), dúvidas (22), estética (17), etapa (26), família (154), inatividade (17), inatividade sexual (17), inexorabilidade (49), isolamento (88), medo (63), memória e esquecimento (62), morte (84), mudanças (54), objetos típicos (59), problemas (18), processo (40), realização (31), reflexão (28), religião e espiritualidade (17), responsabilidade (23), sabedoria e experiência (531), cuidados com a saúde (221), sentimentos positivos (42), sinais físicos (282), sofrimento (59), técnicas de rejuvenescimento (19), tempo (212), trabalho (46), traços negativos de personalidade (44), traços positivos de personalidade (84), tranqüilidade (74), tristeza (91), valorização social, velhice (27), vida (40). Respostas que não puderam ser incluídas nessas categorias por tratarem de conteúdos dispersos e não passíveis de agregação relevante foram desconsideradas da análise, totalizando 205 ocorrências. Assim, a categorização permitiu considerar 3710 respostas de um total de 3915, ou cerca de $95 \%$ das ocorrências.

Uma primeira análise fatorial de correspondências foi realizada a partir de uma tabela indivíduos $\mathrm{X}$ elementos da representação, isto é, 469 X 49. Essa análise visou à identificação dos princípios organizadores mais partilhados pelos participantes. O Phi2 total da análise foi de 7,18. Esse procedimento de análise de correspondências gera um número de fatores equivalente ao de elementos menos 1 . Assim, foram extraídos 48 fatores. $\mathrm{O}$ interesse principal da análise voltava-se para a identificação dos aspectos mais compartilhados dos resultados, então se visou reter apenas os dois primeiros fatores, que mesmo explicando uma pequena porção da inércia $(10,39 \%)$ mostram-se ilustrativos dos principais padrões de resposta. A Figura 1 apresenta a projeção dos dois fatores.

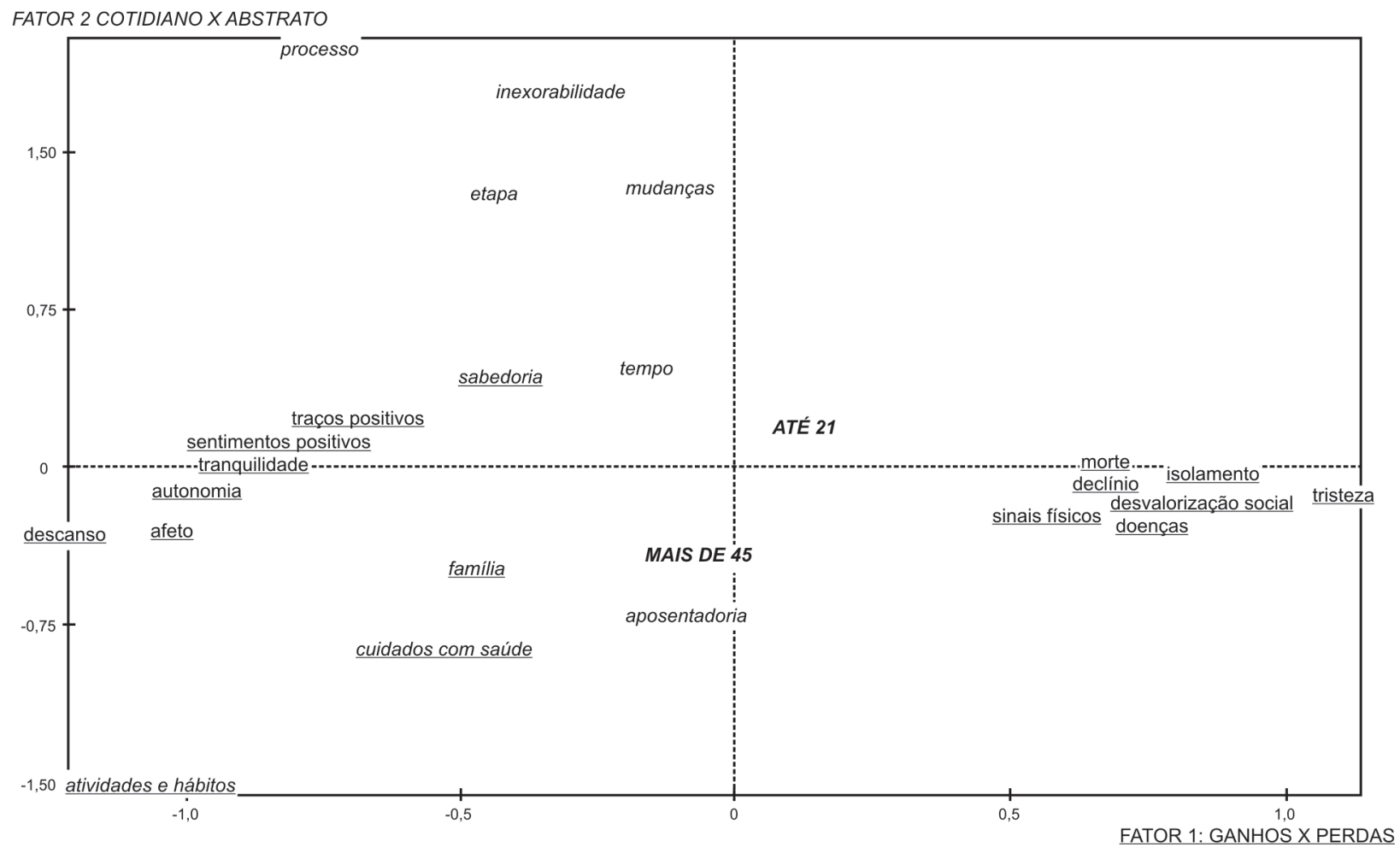

Figura 1. Representação gráfica dos primeiros dois fatores da análise de correspondências indivíduos X elementos.

O primeiro fator opõe perdas e ganhos decorrentes do envelhecimento. Trata-se da contraposição mais marcante e compartilhada dos dados, que não se associa a nenhum grupo específico. No espectro das perdas, o envelhecimento é representado com um período marcado por forte enfraquecimento e desgaste (declínio), tristeza e surgimento de enfermidades, bem como mudanças físicas, desvalorização por parte dos outros, solidão e aproximação da morte. Quanto às vantagens propiciadas ao se envelhecer, há novas atividades e hábitos na rotina, acúmulo de sabedoria e aprendizado, cuidado com a saúde e descanso, tranqüilidade e proximidade familiar.

O segundo fator opõe os participantes mais velhos da amostra, com mais de 45 anos de idade, e os mais jovens, com até 21 anos de idade. Trata-se de um contraste entre aspectos 
mais concretos ligados ao cotidiano e outros abstratos. Os participantes mais próximos da velhice enfatizam elementos ligados ao cotidiano, como a aposentadoria, convívio com a família, relevância de cuidar da saúde e novas ocupações. Já para os mais jovens, o envelhecimento é tratado como uma realidade mais distante, e se enfatiza o processo de envelhecer, ligado à passagem do tempo, levando a uma etapa inevitável com mudanças.

Pode-se observar na Figura 1 que os elementos que tratam de perdas do envelhecimento estão muito próximos no plano, indicando que eles foram mencionados pelas mesmas pessoas. Além disso, como as retas que os ligam à origem formam um ângulo de menos de 90 graus com relação à reta que liga o ponto relativo à modalidade até 21 anos à origem do gráfico, pode-se dizer que também estão em conjunção com esse grupo etário, associando então os participantes mais jovens aos elementos de perdas do envelhecimento. Do lado esquerdo da figura se observa um bloco de elementos ligados a ganhos que se mostra em conjunção com os participantes com mais de 45 anos, e outros mais dispersos, ligados apenas com o segundo fator.

Em seguida, foi realizada uma análise de correspondências baseada numa tabela lexical com os elementos $\mathrm{X}$ as modalidades das variáveis de caracterização sexo, faixa etária, estado civil e região de residência (49 X 15), visando a identificar mais claramente diferenças entre os grupos de modo a situar as ancoragens das representações no espaço intergrupal. O Phi2 total foi de 0,05 . Foram extraídos 11 fatores, dos quais decidiuse por reter 3, que explicaram $56,4 \%$ da inércia total. A Tabela 2 apresenta os três fatores retidos.

O primeiro fator contrapõe os participantes com mais idade, casados ou separados, e os mais jovens, solteiros e do sexo masculino. Os participantes mais velhos abordam uma visão do envelhecimento realista, marcada por benefícios e também desvantagens, mencionando por um lado novas formas de lazer e ocupações, uma vida mais calma, independência e realização frente às conquistas do passado, alterações positivas na personalidade, e por outro uma maior dependência em relação aos outros, preconceito da sociedade, e perdas cognitivas. Já os jovens fazem referência a uma visão mais estereotipada do envelhecimento: processo marcado pelo fim da vida produtiva, em que o idoso, com bastante sabedoria e experiência acumulada, utiliza-se de objetos típicos como bengalas ou cadeiras de balanço e vive próximo da família até a morte.

O segundo fator contrasta viúvos e separados residentes na região sul com participantes das duas faixas etárias mais jovens. Os participantes viúvos e separados falam de uma nova rotina, em que se vive mais intensamente o papel na família, há necessidade de se preocupar com a saúde e surgem outros hábitos e atividades. Envelhecer volta-se para descanso e calma. Já os participantes mais jovens diferenciam-se ao mencionar mudanças fortes ligadas a aspectos negativos e perdas decorrentes do envelhecimento.

Por fim, o terceiro fator opõe mulheres residentes no nordeste do país a homens e participantes separados e da faixa etária de 30 a 45 anos. As mulheres destacam-se por representar o envelhecimento como uma fase ambígua, e diferenciam-se pela menção de sentimentos positivos, afeto e uma busca pela neutralização do processo, por meio de técnicas de rejuvenescimento, como cirurgias plásticas. Os homens e participantes com 30 a 45 anos apresentam uma visão pessimista do envelhecimento, como declínio atrelado à passagem do tempo, caracterizado por desânimo, desprendimento das coisas e perdas cognitivas.

\section{Discussão}

Há dois eixos para nortear a discussão dos resultados obtidos. $\mathrm{O}$ primeiro se refere à representação social do envelhecimento de modo geral, o segundo à diferenciação dos grupos etários e demais agrupamentos formados por variáveis de caracterização em termos de pontos de ancoragem dessa representação.

Em primeiro lugar, cabe esclarecer que o estudo acabou por se caracterizar como um estudo da representação social de jovens e adultos não-idosos sobre o envelhecimento. Como foi uma pesquisa cujo recrutamento foi espontâneo por parte dos participantes de comunidades da Internet, não foi possível garantir uma proporção expressiva de participantes idosos, e por esse motivo eles não foram incluídos na amostra. De todo modo, assim a comparação entre representações de gerações deve ser entendida como um aprofundamento das diferenças entre nãoidosos. Talvez uma menor proporção de idosos com acesso à comunidade Orkut ou mesmo à própria internet inviabilize uma comparação com o tipo de recrutamento do presente estudo. Assim, a coleta de dados presencial provavelmente se apresenta mais adequada para uma caracterização intergrupal mais ampla em nível de grupos geracionais.

Situando o estudo como uma caracterização da representação social de não-idosos sobre envelhecimento, a análise de correspondências baseada na tabela indivíduos por elementos logo apresenta a principal característica da representação social do envelhecimento que está presente na literatura da área: o conflito entre perdas e ganhos (Martins, 2002; Oliveira \& Santos, 2002; Mithidieri \& Tura, 2003; Santos \& Belo, 2000; Teixeira, 2000; Veloz, Nascimento-Schulze, \& Camargo, 1999). A contraposição entre perdas e ganhos refere-se ao contraste entre o enfraquecimento, surgimento de sinais físicos e doenças e discriminação social propiciados pela aproximação do idoso da morte, por um lado; e do outro um reconhecimento de que o idoso acumulou conhecimentos e experiências durante a vida que the conferem respeito, bem como a constatação de que o envelhecimento está associado com uma vida mais tranqüila e de repouso ligada a novas formas de lazer. Esse resultado, reproduzido em outros estudos, confere validade à caracterização do campo realizada, o que permite maior segurança também ao analisar os resultados referentes às comparações entre grupos. A contraposição entre perdas e ganhos é o principal princípio organizador da representação social sobre envelhecimento, e segundo esse critério de segmentação as pessoas adotam posicionamentos mais específicos a seus grupos. Marcante, também, é a ausência de associações de variáveis ilustrativas a respeito desse primeiro fator, o que indica que ele possui generalidade considerável no contexto da amostra. Assim, 
Tabela 2

Análise de correspondências (modalidades X elementos)

\begin{tabular}{|c|c|c|c|c|c|c|c|c|}
\hline \multirow{2}{*}{ Fator } & \multicolumn{4}{|c|}{ Uma realidade próxima } & \multicolumn{4}{|c|}{ Estereótipo do idoso } \\
\hline & Modalidades & Coord. & $\mathrm{CPF}$ & $\operatorname{Cos}^{2}$ & Modalidades & Coord. & $\mathrm{CPF}$ & $\operatorname{Cos}^{2}$ \\
\hline \multirow{13}{*}{$\begin{array}{l}\text { Primeiro, } \\
\text { Autovalor }=0,018 \\
\text { Inércia }=32,4 \%\end{array}$} & Mais de 45 anos & $-0,42$ & 38,8 & 0,82 & Solteiro & 0,11 & 10,5 & 0,81 \\
\hline & Casado & $-0,18$ & 11,7 & 0,61 & Sexo Masculino & 0,12 & 8,3 & 0,39 \\
\hline & Separado & $-0,23$ & 6,7 & 0,27 & Até 21 anos & 0,14 & 7,5 & 0,40 \\
\hline & Elementos & Coord. & $\mathrm{CPF}$ & $\operatorname{Cos}^{2}$ & Elementos & Coord. & $\mathrm{CPF}$ & $\mathrm{Cos}^{2}$ \\
\hline & Ativid.e háb. & $-0,32$ & 16,4 & 0,74 & Família & 0,14 & 4,9 & 0,42 \\
\hline & Dependência & $-0,23$ & 7,1 & 0,64 & Processo & 0,27 & 4,5 & 0,69 \\
\hline & Memória e esq. & $-0,26$ & 6,3 & 0,66 & Morte & 0,17 & 3,6 & 0,56 \\
\hline & Desval. social & $-0,22$ & 5,3 & 0,62 & Direitos & 0,35 & 3,3 & 0,45 \\
\hline & Realização & $-0,30$ & 4,4 & 0,60 & Objetos típicos & 0,19 & 3,3 & 0,50 \\
\hline & Autonomia & $-0,28$ & 4,0 & 0,69 & Aposentadoria & 0,17 & 2,9 & 0,44 \\
\hline & Traços positivos & $-0,16$ & 3,3 & 0,40 & Sabedoria e exp. & 0,06 & 2,8 & 0,40 \\
\hline & Cuid.c.saúde & $-0,09$ & 3,0 & 0,28 & & & & \\
\hline & Tranqüilidade & $-0,15$ & 2,7 & 0,52 & & & & \\
\hline \multirow{13}{*}{$\begin{array}{l}\text { Segundo, } \\
\text { Autovalor }=0,007 \\
\text { Inércia }=12,7 \%\end{array}$} & \multicolumn{4}{|c|}{ Nova rotina } & \multicolumn{4}{|c|}{ Adaptações a perdas } \\
\hline & Viúvo & $-0,25$ & 26,9 & 0,43 & De 22 a 29 anos & 0,10 & 9,8 & 0,26 \\
\hline & Região Sul & $-0,09$ & 17,2 & 0,12 & A té 21 anos & 0,14 & 7,6 & 0,16 \\
\hline & Separado & $-0,23$ & 16,1 & 0,27 & & & & \\
\hline & Elementos & Coord. & $\mathrm{CPF}$ & $\operatorname{Cos}^{2}$ & Elementos & Coord. & $\mathrm{CPF}$ & $\mathrm{Cos}^{2}$ \\
\hline & Descanso & $-0,33$ & 16,8 & 0,66 & Mudanças & 0,16 & 5,2 & 0,33 \\
\hline & Família & $-0,16$ & 15,2 & 0,51 & Declínio & 0,07 & 4,6 & 0,26 \\
\hline & Cuid.c.saúde & $-0,08$ & 5,8 & 0,22 & Traços ne gat. & 0,14 & 3,3 & 0,27 \\
\hline & Ativid.e háb. & $-0,11$ & 5,1 & 0,09 & Sofrimento & 0,12 & 3,2 & 0,22 \\
\hline & Objetos típicos & $-0,13$ & 3,7 & 0,21 & Isolamento & 0,08 & 2,1 & 0,23 \\
\hline & Dúvida & $-0,09$ & 3,4 & 0,33 & Autonomia & 0,13 & 2,1 & 0,14 \\
\hline & Tranqüilidade & $-0,15$ & 2,2 & 0,16 & & & & \\
\hline & \multicolumn{4}{|c|}{ Fase ambígua da vida } & \multicolumn{4}{|c|}{ Enfraquecimento ligado a o tempo } \\
\hline \multirow{15}{*}{$\begin{array}{l}\text { Te rceiro } \\
\text { Autovalor }=0,006 \\
\text { Inércia }=11,3 \%\end{array}$} & Sexo Femi nino & $-0,07$ & 11,7 & 0,25 & De 30 a 45 anos & 0,16 & 29,7 & 0,59 \\
\hline & Região Nordeste & $-0,10$ & 8,1 & 0,14 & Sexo Masculino & 0,09 & 15,3 & 0,25 \\
\hline & & & & & Separado & 0,14 & 7,6 & 0,11 \\
\hline & Elementos & Coord. & $\mathrm{CPF}$ & $\operatorname{Cos}^{2}$ & Elementos & Coord. & $\mathrm{CPF}$ & $\operatorname{Cos}^{2}$ \\
\hline & Etapa & $-0,26$ & 8,0 & 0,21 & Declínio & 0,10 & 10,6 & 0,54 \\
\hline & Sent. positivos & $-0,15$ & 6,8 & 0,33 & Te mp o & 0,09 & 7,6 & 0,42 \\
\hline & Afeto & $-0,15$ & 4,8 & 0,52 & Desprendimento & 0,30 & 4,3 & 0,32 \\
\hline & Problemas & $-0,22$ & 3,9 & 0,53 & Memória e esq. & 0,11 & 3,4 & 0,12 \\
\hline & Téc. rejuvenesc. & $-0,21$ & 3,6 & 0,27 & Aborrecimento & 0,18 & 3,0 & 0,25 \\
\hline & Medo & $-0,11$ & 3,4 & 0,18 & Descanso & 0,13 & 2,7 & 0,10 \\
\hline & Responsabilidade & $-0,18$ & 3,4 & 0,18 & Dependência & $-0,10$ & 2,2 & 0,07 \\
\hline & Desvalor. social & $-0,10$ & 2,9 & 0,12 & & & & \\
\hline & Cuid.c.saúde & $-0,05$ & 2,6 & 0,09 & & & & \\
\hline & Sofrimento & $-0,09$ & 2,3 & 0,14 & & & & \\
\hline & Realizaçã o & $-0,12$ & 2,1 & 0,10 & & & & \\
\hline
\end{tabular}

trata-se de um princípio que parece abarcar o grupo amplo de não-idosos, aproximando as representações de jovens e adultos, e que corrobora os resultados de Martins (2002), em que foram verificadas tão somente diferenças de conteúdos representacionais entre não-idosos e idosos.

Comparando-se os grupos configurados pelas variáveis nominais por meio dos resultados da segunda análise de correspondências, pode-se simular o processo de transformação 
de representações com base na aproximação do envelhecimento entre não-idosos. Ao observar os contrastes entre grupos geracionais, observa-se um maior contraste entre os participantes mais jovens e mais velhos logo no primeiro fator ou princípio organizador. É um contraste entre um envelhecimento altamente estereotipado e uma realidade próxima. O segundo princípio organizador é o que diferencia as duas faixas de participantes mais jovens dos demais; enquanto os participantes mais novos tratam do envelhecimento como mudanças necessárias e decorrentes de um enfraquecimento causado pelo processo, os outros, participantes mais experientes por serem viúvos ou separados, já o abordam como uma nova rotina, um novo cotidiano. E no último fator há uma contraposição entre homens e mulheres, sendo que os participantes da penúltima faixa etária do estudo também estão associados com os do sexo masculino. Os participantes do sexo masculino e com 30 a 45 anos de idade aparentam ter receio em relação ao envelhecimento, associando-o com aspectos degenerativos e à perda da vitalidade na vida ligada à passagem do tempo. As mulheres destacam-se dos homens pela ênfase em aspectos afetivos e uma preocupação com o rejuvenescimento físico.

$\mathrm{O}$ que se observa, se forem comparados os elementos distintivos de cada grupo geracional, é que com o aumento da idade passa-se de uma visão altamente estereotipada dos idosos ligada à adaptação a perdas e um distanciamento abstrato do processo, quando se é mais jovem, para um certo temor em virtude da percepção da aproximação da velhice, marcada por um negativismo extremo. Em seguida, após os 45 anos, ocorre tanto um processo de "desmistificação" quanto de conformismo com a nova realidade que se impõe, e o envelhecimento e velhice tornam-se elementos mais concretos, ligados à rotina cotidiana, aceitos com maior facilidade. Embora o presente estudo tenha tido um delineamento transversal, a comparação intergrupos aparenta simular, nesse caso, resultados que poderiam ser encontrados segundo uma perspectiva longitudinal. Evidentemente, trata-se de uma conjectura que necessitaria da realização de pesquisas para verificação se de fato isso ocorre.

De qualquer modo, os resultados aparentam ser complementados pelo que se verifica na literatura acerca do que viria a ser o próximo grupo etário nessa perspectiva de transformação cronológica da representação social: os idosos. Estudos como os de Veloz, Nascimento-Schulze e Camargo (1999) e Martins (2002) apontam que ocorre uma adaptação frente às novas condições que a velhice proporciona, abrindo-se modos de vida proveitosos e atalhos para contornar os problemas decorrentes do envelhecimento, bem como uma visão das nuances contidas em processos de envelhecimento bem e mal sucedidos. Quando o envelhecimento torna-se um objeto social mais próximo e a velhice se apresenta, há inclusive uma negação por parte dos idosos do papel social destinado pela sociedade e pelos não-idosos às pessoas mais velhas.

Quanto aos aspectos relacionados às diferenças entre sexo, eles foram menos pronunciados na amostra, aparecendo somente na terceira dimensão de uma das análises de correspondência. Ao observar que as participantes do sexo feminino mencionaram, em contraste com os homens, elementos como afeto e técnicas de rejuvenescimento, encontra-se alguma correspondência com a representação de idosas do estudo de Veloz, Nascimento-Schulze e Camargo (1999), que fizeram referência à perda de beleza. Esse aspecto, portanto, pode se mostrar mais compartilhado entre mulheres de diversos grupos geracionais, cabendo sua investigação futura.

O presente estudo consiste numa caracterização dos princípios geradores da representação do envelhecimento, permitindo delinear a supra-estrutura que norteia o posicionamento de representações de grupos específicos no campo representacional. No entanto, ele se pauta principalmente pela identificação entre pontos de ancoragem distintos entre os grupos. Não é possível afirmar acerca de quais seriam os elementos centrais da representação sobre envelhecimento para os grupos geracionais de não-idosos pelo fato de não ter sido realizada uma investigação da estrutura de cada representação, com técnicas como análise prototípica (Vergès, 1992), análise de similitude (Flament, 1981; Vergès \& Bouriche, 2001), técnicas do questionamento (Moliner, 1994) ou esquemas cognitivos de base (Guimelli \& Rouquette, 1992). No contexto brasileiro, ainda não foram realizadas caracterizações conclusivas acerca desse aspecto, cabendo, contudo, mencionar o estudo de Mithidieri e Tura (2003) que aponta que para estudantes de medicina há grande possibilidade de que o elemento sabedoria faça parte do núcleo central da representação, o que poderia talvez ser generalizado a outras pessoas dessa faixa etária. De todo modo, a pertença grupal estudante de medicina provavelmente interfere significativamente nessa caracterização. Na França, Moliner e Vidal (2003), ao estudarem os estereótipos sobre os idosos, chegaram a diagnósticos mais seguros de alguns elementos centrais, como experiência, doenças e tempo livre. Presumivelmente, em alguma extensão esses elementos podem ser transpostos para a representação sobre envelhecimento.

Assim, ainda que estudos sobre os conteúdos da representação social do envelhecimento já existam em alguma quantidade no Brasil, é necessário caracterizar a representação social de modo mais sofisticado estruturalmente, e em especial para verificar possíveis diferenças ou transformações existentes entre grupos geracionais. Além disso, uma caracterização mais precisa em termos da representação também fornece dados acerca dos caminhos por meio dos quais essa representação pode ser alterada, dado que se identificariam os elementos centrais e periféricos. Um outro aspecto que poderia ser verificado refere-se a uma confirmação de uma possível centralidade dos elementos da representação ligados a ganhos; até que ponto esses elementos não estão sendo mencionados por serem "politicamente corretos"? Estudos sobre o contexto de interação dos participantes com a representação permitem uma apreciação das pressões normativas que podem estar incidindo na resposta a questionários ou entrevistas, gerando elementos mascarados da representação ou dando uma falsa centralidade a outros (Flament, Guimelli \& Abric, 2006). A respeito do objeto social envelhecimento, um estudo recente realizado com uma amostra de universitários brasileiros aponta que no caso do grupo etário dos jovens, os resultados referentes à centralidade do elemento sabedoria podem estar sob influência de pressões normativas. No estudo foi verificado que esse elemento perde importância para a representação, enquanto outros de cunho negativo 
tornam-se mais importantes, quando os respondentes fornecem respostas que julgam que "um jovem típico" daria a respeito de envelhecimento (Wachelke \& Lins, 2008).

Finalmente, é importante esclarecer que, embora o presente estudo apresente uma amostra razoavelmente diversificada em termos de grupos etários e regiões de residência, ele não é representativo da população brasileira nem em termos de processo de amostragem nem em termos de composição demográfica. A pesquisa com coleta de dados pela internet está restrita, na maior parte dos casos, ao chamado erro de cobertura (Couper, 2000), isto é, ao fato de que ela faz referência à população com acesso à internet, e não à população em geral. Em estudos sobre representações sociais, isso também ocorre (Wachelke, Camargo, Lins, \& Lima, 2007). E mesmo assim, o estudo ainda está mais limitado aos participantes das comunidades Orkut específicas em que foi divulgado. Percebe-se que a amostra foi composta principalmente por pessoas com alto poder aquisitivo e nível de escolaridade também elevado. Assim, não se trata de um estudo sobre a representação social dos brasileiros sobre envelhecimento, mas sim de uma investigação de uma amostra diversificada de brasileiros sobre esse objeto social. Os resultados são coerentes com a literatura da área, o que aumenta a validade da investigação realizada.

\section{Considerações finais}

O presente estudo buscou caracterizar os princípios organizadores da representação social sobre envelhecimento para uma amostra brasileira. Os resultados, de natureza exploratória, permitem uma antevisão de critérios de organização da representação social do envelhecimento segundo grupos sóciodemográficos, abrindo caminho para estudos futuros sobre o campo dessa representação. Também são fornecidas indicações acerca do processo de evolução da representação conforme o decorrer da passagem de um grupo etário a outro, apresentando o espaço representacional em que se situam as representações de grupos específicos.

Adicionalmente, a coleta de dados por meio da internet consiste numa inovação para estudos de representações sociais. Por permitir acesso a uma amostra diversificada, trata-se de um recurso pertinente, se empregado com controle e consciência de suas limitações em termos de capacidade de generalização.

\section{Referências}

Abric, J.-C. (1998). A abordagem estrutural das representações sociais. In A. S. P. Moreira \& D. C. de Oliveira. (Orgs.), Estudos interdisciplinares de representação social (pp. 27-38). Goiânia: AB.

Araújo, L. F., Carvalho, V. A. M. L., \& Moreira, E. F. (2003). Representações sociais da velhice: um estudo com idosos paraibanos. In Textos Completos da III Jornada Internacional sobre Representações Sociais (pp. 542-556). Rio de Janeiro: Editora da UERJ \& Gráfica MEC.

Araújo, L. F., Coutinho, M. P. L., Barros, D. R., \& Moreira, E. F. (2005). Velhice e instituições geriátricas: um estudo comparativo das representações sociais. In Textos Completos da IV Jornada Internacional sobre Representações Sociais (pp. 2917-2932). João Pessoa: UFPB/Editora Universitária.

Bardin, L. (1977). L'analyse de contenu. Paris: Presses Universitaries de
France.

Brewer, M. B., Dull, V., \& Lui, L. (1981). Perceptions of the elderly: stereotypes as prototypes. Journal of Personality and Social Psychology, 41(4), 656-670.

Carvalho, J. A. M., \& Garcia, R. A. (2003). O envelhecimento da população brasileira: um enfoque demográfico. Cadernos de Saúde Pública, 19(3), 725-733.

Cibois, P. (1983). L'analyse factorielle. Paris: Presses Universitaries de France.

Cibois, P. (1989). Éclairer le vocabulaire des questions ouvertes par les questions fermées: le tableau lexical des questions. Bulletin de Méthodologie Sociologique, 23, 12-23.

Costa, F. G., \& Campos, P. H. F. (2003). Representação social da velhice, exclusão e práticas institucionais. In Textos Completos da III Jornada Internacional sobre Representações Sociais (pp. 589-604). Rio de Janeiro: Editora da UERJ \& Gráfica MEC.

Couper, M. (2000). Web surveys: a review of issues and approaches. Public Opinion Quarterly, 64(4), 464-494.

Doise, W. (1985). Les représentations sociales: définition d'un concept. Connexions, 45, 243-253.

Doise, W., Clemence, A., \& Lorenzi-Cioldi, F. (1992). Représentations sociales et analyses de données. Grenoble: Presses Universitaries de France.

Flament, C. (1981). L'analyse de similitude: une technique pour les recherches sur les representations sociales. Cahiers de Psychologie Cognitive, 1, 375-385.

Flament, C., Guimelli, C., \& Abric, J.-C. (2006). Effets de masquage dans l'expression d'une représentation sociale. Les Cahiers internationaux de psychologie sociale, 69, 15-31.

Gastaldi, A., \& Contarello, A. (2006). Una questione di età: rappresentazioni sociali dell'invecchiamento in giovani e anziani. Ricerche di Psicologia, 20(4), 7-22.

Guimelli, C., \& Rouquette, M. L. (1992). Contribution du modèle associatif des schèmes cognitifs de base à l'analyse structurale des représentations sociales, Bulletin de Psychologie, $\mathrm{N}^{\circ}$ spécial "Nouvelles voies en psychologie sociale", $X L V, 405,196-202$.

Hubbard, A. A. (2007). La rappresentazione sociale dell'anziano: uno studio sulla immagine pubblicitaria attraverso la prospettiva dell'invecchiamento positivo e di successo. Major Thesis in Psychology, Università degli studi di Padova.

Hummert, M. L. (1990). Multiple stereotypes of elderly and young adults: a comparison of structure and evaluations. Psychology and Aging, 5(2), 182-193.

Instituto Brasileiro de Geografia e Estatística (2001). Censo demográfico 2000 Brasília: IBGE. Retirado em 05/02/2007, do IBGE (Instituto Brasileiro de Geografia e Estatística), http://www.ibge.gov.br

Instituto Brasileiro de Geografia e Estatística (2006). Pesquisa Nacional por Amostra de Domicílios 2005. Brasília: IBGE. Retirado em 05/02/2007, do IBGE (Instituto Brasileiro de Geografia e Estatística), http://www.ibge. gov.br

Jodelet, D. (2001). Representações sociais: um domínio em expansão. In D Jodelet (Org.), As representações sociais (pp. 17-44). Rio de Janeiro: Editora da UERJ.

Kessler, E.-M., Rakoczy, K., \& Staudinger, U. M. (2004). The portrayal of older people in prime time television series: the match with gerontological evidence. Ageing and Society, 24(4), 531-552.

Kinsella, K., \& Velkoff, V. A. (2001). An aging world: 2001. Washington: U.S. Government Printing Office.

Kite, M. E., \& Johnson, B. T. (1988). Attitudes toward older and younger adults: a meta analysis. Psychology and Aging, 3(3), 233-244.

Martins, C. R. M. (2002). O envelhecer segundo adolescentes, adultos e idosos usuários do SESC Maringá: um estudo de representações sociais. Dissertação de Mestrado em Psicologia, Universidade Federal de Santa Catarina, Florianópolis.

Mithidieri, O. B., \& Tura, L. F. R. (2003). Os sentidos de idoso para estudantes 
de medicina: subsídios para a seleção de conteúdos curriculares. In Textos Completos da III Jornada Internacional sobre Representações Sociais (pp. 605-619). Rio de Janeiro: Editora da UERJ \& Gráfica MEC

Moliner, P. (1994). Les méthodes de repérage et d'identification du noyau des représentations sociales. In C. Guimelli (Org.), Structures et transformations des représentations sociales (pp. 199-232). Neuchâtel : Delachaux et Niestlé.

Moliner, P., \& Vidal, J. (2003). Stéréotype de la catégorie et noyau de la représentation sociale. Revue Internationale de Psychologie Sociale, 1, $157-175$.

Moscovici, S. (1976). La psychanalyse, son image et son public. Paris: Presses Universitaries de France. (obra originalmente publicada em 1961)

Moscovici, S. (2003). O fenômeno das representações sociais. In S. Moscovici (Org.), Representações sociais: investigações em psicologia social (pp. 29-109). Petrópolis: Vozes.

Neri, A. L. (1991). Envelhecer num país de jovens: significados de velho e velhice segundo brasileiros não-idosos. Campinas: Editora da Unicamp.

Neri, A. L. (2001). Paradigmas contemporâneos sobre o desenvolvimento humano em psicologia e em sociologia. In A. L. Neri (Org.), Desenvolvimento e envelhecimento (pp. 11-37). Campinas: Papirus.

Ng, S. H. (1998). Social psychology in an ageing world: ageism and intergenerational relations. Asian Journal of Social Psychology, 1, 99-116.

Oliveira, A., \& Amâncio, L. (2005). A análise factorial de correspondências no estudo das representações sociais - as representações sociais da morte e do suicídio na adolescência. In A. S. P. Moreira, B. V. Camargo, J. C. Jesuíno, \& S. M. Nóbrega (Orgs.), Perspectivas teórico-metodológicas em representações sociais (pp. 323-362). João Pessoa: UFPB Editora
Universitária

Oliveira, A. M. de, \& Santos, M. F. de S. (2002). O envelhecer: teorias científicas $\mathrm{x}$ teorias populares. Psico, 33(2), 311-326.

Santos, M. F. de S., \& Belo, I. (2000). Diferentes modelos de velhice. Psico, 31(2), 31-48.

Teixeira, M. C. T. V. (2000). Representações sobre a velhice e sobre o self. Revista de Ciências Humanas - Série Especial Temática, 111-139.

Valsiner, J. (2003). Beyond social representations: a theory of enablement. Papers on Social Representations, 12, 7.1-7.16.

Veloz, M. C. T., Nascimento-Schulze, C. M., \& Camargo, B. V. (1999). Representações sociais do envelhecimento. Psicologia Reflexão e Crítica, 12(2), 479-501.

Vergès, P. (1992). L'évocation de l'argent: une méthode pour la définition du noyau central de la représentation. Bulletin de Psychologie, 45(405), 203-209.

Vergès, P., \& Bouriche, B. (2001). L'analyse des données par les graphes de similitude. Auxerre Cedex: Sciences Humaines.

Wachelke, J. F. R., Camargo, B. V., Lins, S. L. B., \& Lima, A. V. (2007). Coleta de dados em ambiente de internet em estudos de representações sociais: comparação estrutural com métodos convencionais. In Resumos da V Jornada Internacional sobre Representações Sociais e III Conferência Brasileira sobre Representações Sociais. Brasília: Editora da UnB.

Wachelke, J. F. R., \& Lins, S. L. B. (2008). Changing masks: a masking effect on young people's social representations on aging? Current Research in Social Psychology, 13(19), 232-242.

Wagner, W. (1998). Sócio-gênese e características das representações sociais. In A. S. P. Moreira, \& D. C. de Oliveira. (Orgs.), Estudos interdisciplinares de representação social (pp. 3-25). Goiânia: AB.

João Fernando Rech Wachelke, doutorando em Psicologia Social e da Personalidade pela Università degli studi di Padova, é mestre em Psicologia pela Universidade Federal de Santa Catarina. Endereço para correspondência: Dipartimento di Psicologia Generale "Vittorio Benussi"; Via Venezia 8; 35131; Padova, Italia. Tel.: 39 393-2469712. E-mail: joao.wachelke@unipd.it / wachelke@yahoo.com

Brigido Vizeu Camargo, doutor em Psicologia Social pela École des Hautes Études en Sciences Sociales, é professor associado no departamento de Psicologia da Universidade Federal de Santa Catarina. E-mail: brigido.camargo@yahoo.com.br

Joana Vieira Hazan é aluna do curso de graduação em Psicologia da Universidade Federal de Santa Catarina. E-mail: joanahazan@hotmail.com

Débora Ribeiro Soares é aluna do curso de graduação em Psicologia da Universidade Federal da Paraíba. E-mail: pikena.debi@gmail.com

Luísa Todeschini Pereira Oliveira, mestranda em Psicologia pela Universidade Federal de Uberlândia, é psicóloga formada pela Universidade Federal de Santa Catarina. E-mail: lu_todeschini@hotmail.com Paula Debiasi Reynaud é aluna do curso de graduação em Psicologia da Universidade Federal de Santa Catarina. E-mail: paula_db@hotmail.com 